\title{
Maternal Stress, Pregnancy Diseases and Child Hyperactivity and Attention Deficit (ADHD)
}

\author{
Castejón 0J*1, Galindez $\mathrm{P}^{1}$, Torres IA ${ }^{1}$, Leal J ${ }^{1}$, Villasmil A ${ }^{1}$, Grumbaum $\mathrm{E}^{\mathbf{1}}$ and Salones de Castejón $\mathrm{M}^{2}$ \\ ${ }^{1}$ Institute of Biological Research, Venezuela \\ ${ }^{2}$ Institute of Clinical Neurosciences, Venezuela
}

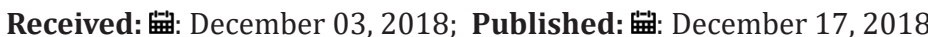

*Corresponding author: Orlando J Castejón, Institute of Biological Research Castejón Foundation, San Rafael Clinical Home, Maracaibo, Venezuela

\begin{abstract}
In the present clinical study the mothers of infant patients with Hyperactivity and Attention Deficit (ADHD) exhibited the followings diseases during pregnancy: Preeclampsia 6\%, hyperemesis gravídarum 4\%, urinary infections $8 \%$, oligohydramnios and loss of amniotic fluid $2 \%$, abnormal uterine bleeding $2 \%$, aging placenta $2 \%$, placental abruption $3 \%$, high blood pressure $8 \%$, diabetes $4 \%$, prepartum depression $7 \%$, postpartum depression 3\%, anxiety, and social problems, such as work, environmental, and conjugal stress. The 100 infant patients examined with Hyperexcitability and Attention Deficit (ADHD) exhibited some of the following associated comorbidities, such as perinatal hypoxia, low weight at birth, behavioural abnormities, anxiety, auto- and hetero aggressivity, autism spectrum disorder, language, learning and hearing disorders, mainly hypoacusia, anorexia or hyperphagia and mental retardation. Social isolation, cognitive deficit, sleeping disorders, talking during sleep, suicidal thoughts, planning and attempts suicidal, parenteral abuse of child. Some non-nervous system co-morbidities, such as pulmonary diseases, and allergic reactions also were found. Some locomotor abnormalities as genus valgo and flat feet were also observed. Preventive interventions suited for the pregnancy period may benefit both maternal and offspring mental health. This line of work should be given high priority on public health research policies mainly in developing countries.
\end{abstract}

Keywords: Maternal Stress; Pregnancy Diseases; Hyperexcitability and Attention Deficit; Clinical Study

\section{Introduction}

Psychosocial stress before and during pregnancy appears to be an independent risk factor for the development of ADHD in their children [1]. The bidirectional relation between family functioning and Attention-Deficit/Hyperactivity Disorder (ADHD) symptoms across the preschool years and primary school has been reported [2]. This is especially the case of families where children have special needs conditions or disorders, like Attention Deficit Hyperactivity Disorder (ADHD) [3]. In addition, this highlights the need to provide support for mothers and fathers who have children with ADHD. Effects of maternal stress on offspring neurodevelopment, cognitive developmental disorders, negative affectivity, difficult temperament and psychiatric disorders are shown in numerous epidemiological and case-control studies. There is not any specific vulnerable period of gestation; prenatal stress effects vary for different gestational ages possibly depending on the developmental stage of specific brain areas and circuits, stress system and immune system. Biological correlates in the prenatally stressed offspring are aberrations in neurodevelopment, neurocognitive function, cerebral processing, functional and structural brain connectivity involving amygdalae and prefrontal cortex, changes in Hypothalamo-Pituitary-Adrenal (HPA)-axis, and autonomous nervous system [4].

Prenatal stress exposure is associated with adverse psychiatric outcomes, including autism and ADHD, as well as locomotor and social inhibition and anxiety-like behaviours in animal offspring. Similarly, maternal immune activation also contributes to psychiatric risk and aberrant offspring behaviour. The mechanisms underlying these outcomes are not clear. Offspring microglia and the pro-inflammatory cytokine Interleukin-6 (IL-6), known to influence microglia, may serve as common mechanisms between prenatal stress and prenatal immune activation. The behavioural effects of prenatal stress in offspring, including increased anxietylike behaviour, decreased sociability and locomotor inhibition, may be related to GABAergic delays, ADHD related lifestyles and resulting comorbidities (e.g., food addiction and obesity, substance abuse, electronic media dependencies and conduct and personality 
disorders). Although ADHD is a neurodevelopmental disorder, its assessment and treatment are also linked to environmental, behavioural and social factors and their interactions [5]. The present paper deals with the relationship between maternal stress, pregnancy diseases and the hyperactivity and attention deficit in children. A clinical study with a correlated neural pathway is described.

\section{Material and Methods}

We have examined 100 infant patients ranging from 3 to 12 years-old with hyperexcitability and attention deficit. Their mothers exhibited maternal stress and different pathological entities during pregnancy. The children were clinically studied at the Clinical Neuroscience Outpatient Clinic of Neuroscience Institute at Clinical Home San Rafael de Maracaibo. They were previously examined from the psychological point of view at our Psychology Department, at CETRO, or at different Public Centre's for Psychology of Maracaibo City.

\section{Results}

In 100 hundred infant ADHD cases studied the mothers exhibited the followings diseases during pregnancy: Preeclampsia, hyperemesis gravídarum, urinary infections, oligohydramnios and loss of amniotic fluid, abnormal uterine bleeding, aging placenta and placental abruption, high blood pressure, diabetes, prepartum depression, postpartum depression, anxiety and social problems, such as work and environmental-related stress, and conjugal stress.

The 100 infant patients examined with Hyperexcitability and Attention Deficit (ADHD) showed some of the following associated comorbidities and risk factors: Preeclampsia 6\%, hyperemesis gravídarum $4 \%$, urinary infections $8 \%$, oligohydramnios and loss of amniotic fluid $2 \%$, abnormal uterine bleeding $2 \%$, aging placenta $2 \%$, placental abruption $3 \%$, high blood pressure $8 \%$, diabetes $4 \%$, prepartum depression $7 \%$, postpartum depression $3 \%$, anxiety, and social problems, such as work, environmental, and conjugal stress. Perinatal hypoxia, prematurity and low weight at birth, behavioural abnormities, anxiety, auto- and hetero aggressivity, autism spectrum disorder, language, learning and hearing disorders, mainly hypoacusia, anorexia or hyperphagia, and mental retardation. Social isolation, cognitive deficit, sleeping disorders, talking during sleep, suicidal thoughts, planning and attempts suicidal, parenteral abuse of child. Some non-nervous system comorbidities, such as pulmonary diseases and allergic reactions also were found. Some locomotor abnormalities as genus valgo and flat feet were also observed.

\section{Discussion}

In the present study we have reported several illnesses related to complications of pregnancy and childbirth, which should be considered in relationship with child Hyperexcitability and Attention Deficit (ADHD). The mechanisms on how these related pregnant diseases should be discussed. We have not found previously published reports on the relationship of hyperemesis gravidarum, oligohydramnios, loss of amniotic liquid, aging placenta and placental abruption with ADHD.

\section{Risk Factors}

Risk factors include familial stressors, physical and psychological and sexual child abuse, anxiety disorders, learning disabilities, abnormal brain development, heritability, and dopamine polymorphisms [6]. Mother hyperemesis gravídarum, oligohydramnios and loss of amniotic fluid, abnormal uterine bleeding during pregnancy, as reported in the present study has not been related thus far as risk factors for child attention deficit. Achievements from various future studies would provide the validity of these pathological entities as risk factors.

\section{Exposure to Maternal Prenatal Stressors}

Epidemiological studies suggest that exposure to prenatal stressors, including malnutrition, Maternal Immune Activation (MIA), and adverse life events, is associated with increased risks of schizophrenia, Autism Spectrum Disorder (ASD) and AttentionDeficit Hyperactivity Disorder (ADHD). The first trimester of pregnancy is particularly a vulnerable period. During this period, the self-renewal of neural stem cells and neurogenesis vigorously occur, and synaptic connections are partially formed in the telencephalon. Disturbance of this neuronal proliferation and migration during the first trimester may underlie the increased susceptibility to these disorders. Epigenetic modifications, such as DNA methylation and histone modification, are critical mechanisms for regulating gene expression. They can be affected by stress and are associated with an increase in susceptibility to schizophrenia and developmental disabilities. An enhanced expression of proinflammatory cytokines, leads to the activation of microglia and the subsequent epigenetic modification of neurons or glia in the offspring [7].

Stress-related symptoms are common in women during pregnancy and are risk factors for neurobehavioral disorders ranging from autism spectrum disorder, attention deficit hyperactivity disorder, and addiction to major depression and schizophrenia. Psychosocial stress before and during pregnancy appears to be an independent risk factor for the development of ADHD in children [1]. The bidirectional relation between family functioning and Attention-Deficit/Hyperactivity Disorder (ADHD) symptoms across the preschool years and primary school has been reported [2]. Therefore, it is important to highlight the importance of potentially offering psychological and social support to mothers who experience stress during pregnancy [8]. Say et al. [10] investigated the shared and non-shared perinatal risk factors for Autism Spectrum Disorders (ASD) and Attention Deficit/ Hyperactivity Disorder (ADHD) in a clinical sample. Additionally, we compared these groups regarding pre/postpartum maternal stress and the duration of breast feeding. Prematurity of the neonate and maternal stress/depressive mood in pregnancy were common risk factors shared by ASD and ADHD. Postpartum maternal depressive mood may be more specific to ASD, while shorter duration of breastfeeding may be related to ADHD. The effect of prenatal stressful life events on ADHD symptoms in offspring may depend on the timing of prenatal stress and may vary according to the sex of the offspring [11]. 
On the contrary, ADHD can impair mother mental health by inducing stress and this issue has important clinical and treatment implications. Specific treatment programs should be designed and implemented for mothers of ADHD children to reduce stress among them and therefore, improve their mental health status. Mothers of ADHD children had also more stress compared with mothers of normal children $[12,13]$ highlight the complex interplay between prenatal stress exposure, associated changes in miRNA expression and DNA methylation in placenta and brain and possible links to greater risks of schizophrenia, attention deficit hyperactivity disorder, autism, anxiety- or depression-related disorders later in life. Based on existing evidence, we propose that prenatal stress, through the generation of epigenetic alterations, becomes one of the most powerful influences on mental health in later life. Franc et al. [14] analyse the relationship between ADHD and attachment processes. ADHD is described as a multifactorial disease, with a well-studied genetic vulnerability, and early environmental factors also playing an important role in the development and course of the disorder. Current aetiological models emphasize interaction between genes and environment.

First, clinical findings emphasize similitude between both disorders; emotional dysregulation is an important feature in reactive attachment disorder as well as in ADHD. Emotion regulation is highly related to attachment security in young children and could play a part in the development of early attention processes. Some perinatal factors, such as smoking during pregnancy or prematurity, have been shown to increase the risk of hyperactive symptoms in children. These variables may also be associated with a higher risk of impaired early interactions. Recent animal studies have raised interest in the role of prenatal stress in the emotional and behavioural development of the offspring, particularly as regards vulnerability to stress. Epigenetic mechanisms may be involved in durable alterations of the hypothalamo-pituitary-adrenergic axis.

\section{Maternal Pathology and Perinatal Hypoxia}

Effects of maternal stress on offspring neurodevelopment, cognitive development, negative affectivity, difficult temperament and psychiatric disorders are shown in numerous epidemiological and case-control studies. There is not any specific vulnerable period of gestation; prenatal stress effects vary for different gestational ages possibly depending on the developmental stage of specific brain areas and circuits, stress system and immune system. Biological correlates in the prenatally stressed offspring are aberrations in neurodevelopment, neurocognitive function, cerebral processing, functional and structural brain connectivity involving amygdalae and prefrontal cortex, changes in Hypothalamo-Pituitary-Adrenal (HPA)-axis, and autonomous nervous system [4]. Epidemiological studies suggest that exposure to prenatal stressors, including malnutrition, Maternal Immune Activation (MIA) and adverse life events, is associated with increased risks of schizophrenia, Autism Spectrum Disorder (ASD), and Attention-Deficit Hyperactivity Disorder (ADHD). The first trimester of pregnancy is particularly a vulnerable period. During this period, the self-renewal of neural stem cells and neurogenesis vigorously occur, and synaptic connections are partially formed in the telencephalon.
Disturbance of this neuronal proliferation and migration during the first trimester may underlie the increased susceptibility to these disorders. Epigenetic modifications, such as DNA methylation and histone modification, are critical mechanisms for regulating gene expression. They can be affected by stress and are associated with an increase in susceptibility to schizophrenia and developmental disabilities. An enhanced expression of proinflammatory cytokines, leads to the activation of microglia and the subsequent epigenetic modification of neurons or glia in the offspring [7]. Very preterm, extremely preterm, very low birth weight, and extremely low birth weight newborns seem to have a higher risk of later AttentionDeficit/Hyperactivity Disorder (ADHD), the magnitude of the risk is not well-defined. There is an increased risk of ADHD diagnosis and symptomatology compared with controls [15].

\section{The Critical Role of Placenta}

In our study we have found aging placenta and placental abruption in ADHD child's. The placenta has a critical role in the deleterious and sex-specific effects of maternal stress and other fetal exposures on the developing brain. Stress-induced perturbations of the maternal milieu are conveyed to the embryo via the placenta, the maternal-fetal intermediary responsible for maintaining intrauterine homeostasis. Disruption of vital placental functions can have a significant impact on fetal development, including the brain, outcomes that are largely sex-specific [16]. Prenatal stress-induced increases in placental inflammation and offspring hyperactivity are male-specific and ameliorated by maternal antiinflammatory treatment. Involve pathways common to both stress and immune responses at the maternal-fetal interface. Maternal stress induced activation of immune pathways within the placenta, the sex-specific maternal-fetal intermediary, may contribute to prenatal stress programming effects on the offspring.

Stress-induced locomotor hyperactivity, a hallmark of dopaminergic dysregulation, dopamine D1 and D2 receptors is altered by Early Prenatal Stress (EPS) in males. These studies support an important interaction between maternal stress and a proinflammatory state in the long-term programming effects of maternal stress [17]. Morphological changes of placental aging are common and seem to have no effect on fetus and on Doppler flow of the umbilical and uterine arteries, provided these are not highrisk pregnancies and placental changes are not infarction, villitis or severe structural or localization anomaly [18].

\section{Endophenotypes and ADHD}

The thalamo-cortico-striatal circuits appear as the common anatomic substrate for all causal models for ADHD. Thalamocortico-striatal circuits and limbic system are recognized as the anatomic and functional substrate for all causal neuro-cognitive models for ADHD [19]. In this context, any electrophysiological, behavioural, neuro-humoral or anatomic marker related with functions commanded by such system (mainly executive functions and reward functions) could be a promising endophenotype for ADHD. In recent years, numerous molecular genetic studies have been published to investigate susceptibility loci for ADHD, and its candidate genes. including linkage studies, candidate-gene 
association studies, genome-wide association studies and genomewide copy number variation studies, with a special focus on general patterns of study design and common sample features; 2 candidate genes for ADHD have been systematically evaluated in three ways for better utilization. The thorough summary of the achievements from various studies will provide an overview of the research status of molecular genetics studies for ADHD [20].

\section{Proinflammatory Cytokines}

Proinflammatory cytokines Interleukin-1, Interleukin-6 (IL1, IL-6) and Tumour Necrosis Factor Alpha (TNF alpha), the key mediators of neuroimmune interactions, are the common pathogenic part of various kinds of the perinatal pathology leading to severe neurological and mental diseases [21]. Cytokines are alternative possible mediators. An additional explanation is that stress or anxiety causes increased transfer of maternal cortisol across the placenta to the fetus. The placenta plays a crucial role in moderating fetal exposure to maternal factors and presumably in preparing the fetus for the environment in which it is going to find itself. There is some evidence in both rat models and in humans that prenatal stress can reduce placental 11 $\beta$-HSD2, the enzyme which metabolizes cortisol to inactive cortisone. The level of cortisol in the amniotic fluid, surrounding the baby in the womb, has been shown to be inversely correlated with infant cognitive development. However, several other biological systems are likely to be involved. Serotonin is another possible mediator of prenatal stress induced programming effects on offspring neurocognitive and behavioural development. The role of epigenetic changes in mediating alterations in offspring outcome following prenatal stress is likely to be important and starting to be explored [22].

Higher levels of cortisol exposure are also hypothesized to underlie the mechanism through which maternal stress may disrupt fetal development. These intriguing and suggestive results demonstrate that this line of work should be given high priority, and they set the stage for additional research moving forward [8].

\section{Maternal Mental Diseases and ADHD}

In our study we have reported pre- and postpartum depression and anxiety. Maternal depressive symptoms throughout pregnancy are associated with increased ADHD symptomatology in young children. Maternal depressive symptoms after pregnancy add to, but only partially mediate, the prenatal effects. Preventive interventions suited for the pregnancy period may benefit both maternal and offspring mental health [23]. We have also reported high blood pressure in our patients with or without associate's eclampsia. Hypertensive Disorders of Pregnancy (HDPs),that is chronic hypertension, gestational hypertension, pre-eclampsia (de novo or superimposed on chronic hypertension) and white coat hypertension, affect approximately 5\%-15\% of pregnancies. HDP exposure has been linked to an increased risk of autism spectrum disorder, attention deficit/hyperactivity disorder and other neurodevelopmental disorders in children [24].

In relationship with sleep disturbances in children, Morales Muñoz et al. [25] have studies have been focused on maternal prenatal depression and/or anxiety as potential risk factors for sleep problems in childhood, whereas other relevant psychological factors during pregnancy have not received as much attention. They have examined the effect of several psychiatric maternal risk factors during pregnancy (i.e. symptoms of anxiety, depression, insomnia, alcohol use, seasonality, attention deficit and hyperactivity disorder and/or stressful life events) on the onset of some sleep problems related to sleep quality and sleep practices in 3-month-old infants.

\section{Maternal Urinary Tract Infection and ADHD}

In our study we have reported a high incidence of maternal urinary tract infection. Smoking in pregnancy, maternal urinary tract infection, being induced, and experiencing threatened preterm labour increase the risk of ADHD, Maternal genitourinary infection and preeclampsia was associated with significantly increased odds of ADHD [19,26].

\section{The Maternal Metabolic State, Obesity and Diabetes}

Prenatal exposure to metabolic disturbances is associated with increased risk of offspring neurodevelopmental impairment and autism spectrum disorder, while little is known about the joint effect of maternal obesity and diabetes. Maternal Pre-Gestational Diabetes Mellitus (PGDM) combined with severe maternal obesity markedly increases the risk of several children's psychiatric and mild neurodevelopmental disorders [27,28]. Prenatal maternalvery severe obesity is a strong predictor of increased neuropsychiatric problems in early childhood [29]. Maternal low Socioeconomic Status (SES) heighten the risk for childhood ADHD [30]. According to Instanes et al. [28], several maternal somatic diseases with immune components were found to increase the risk of ADHD in offspring. The associations could involve several causal pathways, including common genetic predisposition and environmental factors, and increased insight into the mechanisms behind these relationships could enhance our understanding of the etiology of ADHD.

If a mother is stressed while pregnant, her child is substantially more likely to have emotional or cognitive problems, including an increased risk of attentional deficit/hyperactivity, anxiety, and language delay [29]. Animal models suggest that activity of the stress-responsive Hypothalamic-Pituitary-Adrenal (HPA) axis and its hormonal end-product cortisol are involved in these effects in both mother and offspring. Maternal cortisol responses to stress decline over the course of pregnancy, and earlier in pregnancy, it is possible that the effects of maternal anxiety and stress on the developing fetus and child are moderated by other factors such as a maternal diet (e.g., protein load). Previous research indicates that children from violent marriages are more likely to suffer from conduct problems and/or anxiety disorders than children from nonviolent, satisfactory marriages [30].

\section{Coexisting Hyperactivity, Attention Deficit and Autism Spectrum Disorder}

Over the years, several authors have reported symptoms of Attention Deficit Hyperactivity Disorder (ADHD) in patients with 
Autism Spectrum Disorders (ASD) [31,32]. The high presence of Attention Deficit Hyperactivity Disorder (ADHD) in Autism Spectrum Disorder (ASD) has been acknowledged in the Diagnostic and statistical manual of mental disorders, fifth edition, thus allowing the diagnosis of both disorders [33]. According to Kerekes et al. and Muskens et al. [34], the associations between neurodevelopmental disorders and personality are at least partly due to genetic effects influencing both conditions.

\section{Mother and Child Anxiety Disorders}

We have above described ADHD in children and mothers with fear and nervousness and mood disorders. These children often reject the clinical examination. Separation anxiety was observed in those children whose parents have migrated from their home country or being separated as conjugal partners. According to Christopher et al. [35], ADHD children have more active frontal, temporal, parietal, and occipital lobes during concentration than children diagnosed with anxiety in the same brain areas.

\section{Physical, Psychological and Sexual Child Abuse}

In a few cases the mother has expressed physical, psychological and sexual child abuse of her children. The separation of conjugal relations and the legal process against the father is frequently initiated. This implies the need for greater accuracy in the differential diagnosis, as until a few years ago, post-traumatic stress disorder was considered the reference symptoms for this type of case. Thus, it is necessary to define and conceptualize an increasingly broad and detailed world of sequels and consequences, where ADHD may be related to the psychological damage suffered by child abuse [36].

\section{Abnormal Auditory Processing Pathways in ADHD}

Diagnostic audiological test performed in children showed remarkable differences between the results of behavioural audiometry and those of auditory evoked response audiometry. The results of neuropsychological evaluation showed attention disorders. It is possible that a response desynchronization in the auditory pathway may exist in these disorders [37]. Endogenous evoked potentials especially the $\mathrm{P} 300$ wave, related to the processes of selective attention and sensory elaboration of discriminatory stimuli, have been frequently used. used. It has been suggested that measures of Auditory Processing (AP) are sensitive measures of attention resulting in the high comorbidity of Auditory Processing Disorder (APD) and Attention Deficit/Hyperactivity Disorder (ADHD) [38]. Neuroscience research on auditory processing pathways and their behavioural and electrophysiological correlates has taken place largely outside the field of clinical neuropsychology. Deviations and disruptions in auditory pathways in children and adolescents result in a well-documented range of developmental and learning impairments frequently referred for neuropsychological evaluation [39].

\section{Child Language Impairment}

The neural underpinnings of vocal emotion processing deficits in ADHD have yet to be characterized. The study of Gau, et al.[40] provides the first evidence linking ADHD to atypical neural activity during the early perceptual stages of vocal anger processing. There is little information about processing of nonspeech and speech stimuli at the subcortical level in individuals with attention deficit Hyperactivity Disorder (ADHD). The Auditory Brainstem Response (ABR) provides information about the function of the auditory brainstem pathways. There is a common dysfunction in the processing of click and speech stimuli at the brainstem level in children with suspected ADHD [41].

\section{Learning and Memory Deficit}

In the present paper we have found a subgroup featured by learning and memory deficit and dysgraphia. Children suffering from Attention-Deficit Hyperactivity Disorder (ADHD) often also display impaired learning and memory. Previous research has documented aberrant reward processing in ADHD as well as impaired sleep-dependent consolidation of declarative memory. Studies of ADHD children point toward an essential contribution of prefrontal cortex to the preferential consolidation of declarative memory during Slow Wave Sleep (SWS) [42]. According to Gau et al. [43], impaired school functioning and altered white matter integrity in front striatal networks have been associated with Attention-Deficit/Hyperactivity Disorder (ADHD). Deficits and ADHD symptoms may be the mediating mechanisms for this association. These patients are characterized by a prefrontal hypoactivity. Therefore, the Authors formulate the hypothesis that children with ADHD benefit from sleep with respect to procedural memory more than healthy children.

Children with ADHD showed an improvement in motor skills after sleep compared to the wake condition. These data suggest that sleep in ADHD normalizes deficits in procedural memory observed during daytime [44]. Research initially supported the theory that deficits in Executive Function (EF) underlie the core neuropsychological deficit of Attention-Deficit/Hyperactivity Disorder (ADHD), particularly deficits in working memory and inhibitory control arising from dysfunction in the prefrontal cortex. However, recent findings have called the EF deficit theory of ADHD into question, and research on the specificity of both direct and indirect measures of $\mathrm{EF}$ has not yielded promising results. EF measures can, in light of the most current science, still remain a useful part of a neuropsychological test battery [45]. Boys with ADHD comorbid LD show deficits in overall memory function and long-term memory while short-term memory is partially damaged. Impairment in immediate memory is not detected [46].

\section{Eating Disorders}

In our study most, patients exhibited eating disorders mainly, hyperphagia. George et al. [47] estimate that little is known about the neurocognitive mechanisms that mediate the association between Attention Deficit/Hyperactivity Disorder (ADHD) and Eating Disorders (ED). The literature has suggested that eating disorders and ADHD could be explained as different expressions of common biological underpinnings. The Authors suspect there is a common neuropsychological pathway that implicates selfregulation deficits. 


\section{Allergic Diseases in Children with Attention Deficit Hyperactivity Disorder}

A recent meta-analysis show that children with ADHD are more likely to have asthma, allergic rhinitis, atopic dermatitis, and allergic conjunctivitis than their counterparts (meta-analysis show that children with ADHD are more likely to have asthma, allergic rhinitis, atopic dermatitis, and allergic conjunctivitis than their counterparts $[48,49]$. Early food allergy is associated with ADHD in school-age children. Early food allergy and respiratory allergy symptoms independently and synergistically contributed to higher risk of ADHD [50].

\section{Physical, Psychological and Sexual Child Abuse}

In a few cases the mother has expressed physical, psychological and sexual child abuse of her children. The separation of conjugal relations and the legal process against the father is frequently initiated. This implies the need for greater accuracy in the differential diagnosis, as until a few years ago, post-traumatic stress disorder was considered the reference symptoms for this type of case. Thus, it is necessary to define and conceptualize an increasingly broad and detailed world of sequels and consequences, where ADHD may be related to the psychological damage suffered by child abuse [36].

\section{Sleep Dysfunction and ADHD}

ADHD children could present sleep problems such as agitated sleep, sleep-onset difficulties, low arousal threshold during the night. Such sleep difficulties have been shown to occur more frequently in children with severe diurnal ADHD symptoms. Prevalence and physiopathology of sleep disorders including awakening mechanisms (micro-arousals, arousal threshold) from sleep yet need to be clarified in ADHD children. Excessive nocturnal motricity could be the expression of a monoaminergic dysfunction previously reported in ADHD and could lead to new therapeutic gateways as well as hypovigilance. Sleep problems in children with ADHD are commonly transient, but in a subgroup, they are characterized as persistent. Early preventive/intervention strategies should target children at risk of persistent sleep problems. Hvolby presents a conceptual model of the modes of interaction: ADHD may cause sleep problems as an intrinsic feature of the disorder; sleep problems may cause or mimic ADHD; ADHD and sleep problems may interact, with reciprocal causation and possible involvement of comorbidity; and ADHD and sleep problems may share a common underlying neurological etiology, Among the mediators that may participate in ADHD, melatonin is thought to regulate circadian rhythms, neurological function and stress response. Melatonin was higher Predominantly in Hyperactive-Impulsive/Conduct Disordered children (PHI/CD) of the ADHD subtype. A differential cerebral melatonin metabolization after methylphenidate may underlie some of the clinical benefit.

\section{Aggressive Behaviour and ADHD}

Anxiety and Attention-Deficit/Hyperactivity (ADH) problems are common in adolescence, often co-occur, and are characterized by high heterogeneity in their phenotypic expressions. Although it is known that anxiety and $\mathrm{ADH}$ problems correlate, the relationships between subtypes of anxiety and ADH problems have been scarcely investigated. The association between ADH problems and anxiety could be entirely attributed to attention problems, not hyperactivity/impulsivity.

\section{Neural Correlates of ADHD}

Hyperexcitability and Attention Deficit (ADHD) is characterized by multiple functional and structural neural network abnormalities including most prominently front-striatal, but also front-parietotemporal, front-cerebellar and even front-limbic networks. Evidence from longitudinal structural imaging studies has shown that ADHD is characterized by a delay in structural brain maturation [51]. Attention-Deficit/Hyperactivity Disorder (ADHD) research has long focused on the dopaminergic system's contribution to pathogenesis, although the results have been inconclusive. However, the involvement of the noradrenergic system, which modulates cognitive processes, such as arousal, working memory, and response inhibition, all of which are typically affected in ADHD [52]. Dopamine and norepinephrine are the main neurotransmitters involved in the pathophysiology of ADHD. Patients showed significantly reduced dorsal caudate functional connectivity with the superior and middle prefrontal cortices as well as reduced dorsal putamen connectivity with the parahippocampal cortex [53].

According to Gau et al. [43], impaired school functioning and altered white matter integrity in front striatal networks have been associated with Attention-Deficit/Hyperactivity Disorder (ADHD). Deficits and ADHD symptoms may be the mediating mechanisms for this association. These patients are characterized by a prefrontal hypoactivity. Therefore, the Authors formulate the hypothesis that children with ADHD benefit from sleep with respect to procedural memory more than healthy children [54-64]. Children with ADHD showed an improvement in motor skills after sleep compared to the wake condition. These data suggest that sleep in ADHD normalizes deficits in procedural memory observed during daytime [44]. Research initially supported the theory that deficits in Executive Function (EF) underlie the core neuropsychological deficit of Attention-Deficit/Hyperactivity Disorder (ADHD), particularly deficits in working memory and inhibitory control arising from dysfunction in the prefrontal cortex. However, recent findings have called the EF deficit theory of ADHD into question, and research on the specificity of both direct and indirect measures of EF has not yielded promising results. EF measures can, in light of the most current science, still remain a useful part of a neuropsychological test battery [45]. Boys with ADHD comorbid LD show deficits in overall memory function and long-term memory while short-term memory is partially damaged. Impairment in immediate memory is not detected [46]. Thalamo-cortico-striatal circuits and limbic system are recognized as the anatomic and functional substrate for all causal neuro-cognitive models for ADHD [19].

\section{Concluding Remarks}

In the present clinical study, the mothers exhibited the followings diseases during pregnancy: Preeclampsia, hyperemesis 
gravídarum, urinary infections, oligohydramnios, loss of amniotic fluid, abnormal uterine bleeding, aging placenta, placental abruption, high blood pressure, diabetes, prepartum depression, postpartum depression, anxiety, and social problems, such as work and environmental-related stress, and conjugal stress. The 100 infant patients examined with hyperexcitability and Attention Deficit (ADHD) exhibited some of the following associated comorbidities, such as perinatal hypoxia, low weight at birth, behavioural abnormities, anxiety, auto- and hetero aggressivity, autism spectrum disorder, language, learning and hearing disorders, mainly hypoacusia, anorexia or hyperphagia, and mental retardation. Social isolation, cognitive deficit, sleeping disorders, perinatal, talking during sleep, suicidal thoughts, planning and attempts suicidal, parenteral abuse of child. Some non-nervous system comorbidities, such as pulmonary diseases and allergic reactions also were found. Some locomotor abnormalities as genus valgo and flat feet were also observed. Preventive interventions suited for the pregnancy period may benefit both maternal and offspring mental health. This line of work should be given high priority in public health research policies mainly at under developing countries.

\section{Acknowledgement}

This investigation has been carried out through a subvention obtained from Castejón Foundation, and the administrative and logistic support of Biological Research Institute, Faculty of Medicine, Zulia University, and Home Clinic San Rafael de Maracaibo.

\section{References}

1. Okano L, Ji Y, Riley AW, Wang X (2018) Maternal psychosocial stress and children's ADHD diagnosis: a prospective birth cohort study. J Psychosom Obstet Gynaecol 23: 1-9.

2. Lai KYC, Ma JLC, Xia LLL (2018) Multifamily therapy for children With ADHD in Hong Kong: The different impacts on fathers and mothers. J Atten Disord 1: 1087054718756195.

3. Muñoz Silva A, Lago Urbano R, Sanchez Garcia M, Carmona Márquez J (2017) Child/Adolescent's ADHD and parenting stress: The mediating role of family Impact and conduct problems. Front Psychol 8: 2252.

4. Van den Bergh BRH, van den Heuvel MI, Lahti M, Braeken M, de Rooij SR, et al. (2017) Prenatal developmental origins of behaviour and mental health: The influence of maternal stress in pregnancy. Neurosci Biobehav Rev 1: 3-15.

5. Gumusoglu SB, Fine RS, Murray SJ, Bittle JL, Stevens HE (2017) The role of IL-6 in neurodevelopment after prenatal stress. Brain Behav Immun 65: $274-283$.

6. Ota T, Iida J, Nakanishi Y, Sawada S, Matsuura H, et al. (2015) Increased prefrontal hemodynamic change after atomoxetine administration in pediatric attention deficit/hyperactivity disorder as measured by nearinfrared spectroscopy. Psychiatry Clin Neurosci 69(3): 161-170.

7. Udagawa J, Hino K (2016) Impact of maternal stress in pregnancy on brain function of the offspring. Nihon Eiseigaku Zasshi 71(3): 188-194.

8. Grizenko N, Fortier MÈ, Gaudreau Simard M, Jolicoeur C, Joober R (2015) The effect of maternal stress during pregnancy on IQ and ADHD symptomatology. J Can Acad Child Adolesc Psychiatry 24(2): 92-99.

9. Natalie Grizenko, Marie Ève Fortier, Mathilde Gaudreau Simard, Claude Jolicoeur, Ridha Joober (2015) The effect of maternal stress during pregnancy on IQ and ADHD symptomatology. J Can Acad Child Adolesc Psychiatry 24(2): 92-99.
10. Say GN, Karabekiroğlu K, Babadağı Z, Yüce M (2016) Maternal stress and perinatal features in autism and attention deficit/hyperactivity disorder. Pediatr Int 58(4): 265-269.

11. Zhu P, Hao JH, Tao RX, Huang K, Jiang XM, et al. (2015) Sex-specific and time-dependent effects of prenatal stress on the early behavioural symptoms of ADHD: A longitudinal study in China. Eur Child Adolesc Psychiatry 24(9): 1139-1147.

12. Samiei M, Daneshmand R, Keramatfar R, Khooshabi K, Amiri N, et al. (2015) Attention deficit hyper activity Disorder (ADHD) and stress: A mutual relationship between children and mothers. Basic Clin Neurosci 6(2): 113-121.

13. Babenko O, Kovalchuk I, Metz GA (2015) Stress-induced perinatal and transgenerational epigenetic programming of brain development and mental health. Neurosci Biobehav Rev 48: 70-91.

14. Franc N, Maury M, Purper Ouakil D (2009) ADHD and attachment processes: are they related? Encephale 35(3): 256-261.

15. Franz AP, Bolat GU, Bolat H, Matijasevich A, Santos IS, et al. (2017) Attention-deficit/hyperactivity disorder and very preterm/very low birth weight: A meta-analysis. Paediatrics 141(1).

16. Bronson SL, Bale TL (2016) The placenta as a mediator of stress effects on neurodevelopmental reprogramming. Neuropsychopharmacology. 41(1): 207-218.

17. Bronson SL, Bale TL (2014) Prenatal stress-induced increases in placental inflammation and offspring hyperactivity are male-specific and ameliorated by maternal anti-inflammatory treatment. Endocrinology 155(7): 2635-2646.

18. Kara SA, Toppare MF, Avşar F, Caydere M (1999) Placental aging, fetal prognosis and fetomaternal Doppler indices. Eur J Obstet Gynecol Reprod Biol 82(1): 47-52.

19. Castejón OJ (2018) Neural correlates of cortico-thalamic-stratiatal and limbic pathway in ADHD. Venezuelan J Neurosci 1: 120.

20. Li Z, Chang SH, Zhang LY, Gao L, Wang J (2014) Molecular genetic studies of ADHD and its candidate genes: a review. Psychiatry Res 219(1): 1024.

21. Zubarev OE, Klimenko VM (2011) Elevation of proinflammatory cytokines level at early age as the risk factor of neurological and mental pathology development. Ross Fiziol Zh Im I M Sechenova 97(10): 10481059.

22. Glover V (2015) Prenatal stress and its effects on the fetus and the child: Possible underlying biological mechanisms. Adv Neurobiol 10: 269-283.

23. Wolford E, Lahti M, Tuovinen S, Lahti J, Lipsanen J, et al. (2017) Maternal depressive symptoms during and after pregnancy are associated with attention-deficit/hyperactivity disorder symptoms in their 3- to 6-yearold children. PLoS One 12(12): e0190248.

24. Maher GM, O Keeffe GW, Kenny LC, Kearney PM, Dinan TG, et al. (2017) Hypertensive disorders of pregnancy and risk of neurodevelopmental disorders in the offspring: A systematic review and meta-analysis protocol. BMJ Open 7(10): e018313.

25. Morales Muñoz I, Saarenpää Heikkilä O, Kylliäinen A, Pölkki P, Porkka Heiskanen T, et al. (2018) The effects of maternal risk factors during pregnancy on the onset of sleep difficulties in infants at 3 months old. J Sleep Res 27(5): e12696.

26. Mann JR, McDermott S (2011) Are maternal genitourinary infection and pre-eclampsia associated with ADHD in school-aged children? J Atten Disord 15(8): 667-673.

27. Nomura Y, Marks DJ, Grossman B, Yoon M, Loudon H, et al. (2012) Exposure to gestational diabetes mellitus and low socioeconomic status: effects on neurocognitive development and risk of attention-deficit/ hyperactivity disorder in offspring. Arch Pediatr Adolesc Med 166(4): 337-343. 
28. Instanes JT, Halmøy A, Engeland A, Haavik J, Furu K, et al. (2017) Attention-deficit/hyperactivity disorder in offspring of mothers with inflammatory and immune system diseases. Biol Psychiatry 81(5): 452 459.

29. Talge NM, Neal C, Glover V (2007) Antenatal maternal stress and longterm effects on child neurodevelopment: how and why? J Child Psychol Psychiatry 48(3-4): 245-261.

30. Jouriles EN, Barling J, O Leary KD (1987) Predicting child behaviour problems in maritally violent families. J Abnorm Child Psychol 15(2): 165-173.

31. Lamanna AL, Craig F, Matera E, Simone M, Buttiglione M, et al. (2017) Risk factors for the existence of attention deficit hyperactivity disorder symptoms in children with autism spectrum disorders. Neuropsychiatr Dis Treat. 13: 1559-1567.

32. Mansour R, Dovi AT, Lane DM, Loveland KA, Pearson DA (2017) ADHD severity as it relates to comorbid psychiatric symptomatology in children with Autism Spectrum Disorders (ASD). Res Dev Disabil 60: 52-64.

33. Berenguer Forner C, Miranda Casas A, Pastor Cerezuela G, Roselló Miranda R (2015) Comorbidity of autism spectrum disorder and attention deficit with hyperactivity. A review study. Rev Neurol 60(Suppl) 1: S37-S43.

34. Muskens JB, Velders FP, Staal WG (2017) Medical comorbidities in children and adolescents with autism spectrum disorders and attention deficit hyperactivity disorders: A systematic review. Eur Child Adolesc Psychiatry 26(9): 1093-1103.

35. Christopher S Martin, Mitch Earleywine, Timothy C Blackson, Ralph E Tarter (1994) Aggressivity, inattention, hyperactivity, and impulsivity in boys at high and low risk for substance abuse. J Abn Child Psychol 22(2): 177-203.

36. Cornellà Canals J, Juárez López JR (2014) Attention-deficit hyperactivity disorder symptoms and their relationship with child abuse: Predictor and consequence. Ann Pediatr (Barc) 81(6): 398-395.

37. Chronaki G, Benikos N, Fairchild G, Sonuga Barke EJ (2015) Atypical neural responses to vocal anger in attention-deficit/hyperactivity disorder. J Child Psychol Psychiatry 56(4): 477-487.

38. Jafari Z, Malayeri S, Rostami R (2015) Subcortical encoding of speech cues in children with attention deficit hyperactivity disorder. Clin Neurophysiol 126(2): 325-332.

39. Wiesner CD, Molzow I, Prehn Kristensen A, Baving L (2017) Sleepdependent consolidation of rewarded behavior is diminished in children with disorder and a comorbid disorder of social behavior. Front Psychol 8: 167-169.

40. Gau SS, Tseng WL, Tseng WY, Wu YH, Lo YC (2014) Association between microstructural integrity of frontostriatal tracts and school functioning: ADHD symptoms and executive function as mediators. Psychol Med 30: $1-15$.

41. Prehn Kristensen A, Molzow I, Munz M, Wilhelm I, Müller K, et al (2011) Transcranial oscillatory direct current stimulation during sleep improves declarative memory consolidation in children with attentiondeficit/hyperactivity disorder to a level comparable to healthy controls. Brain Stimulation 7(6): 793-799.

42. Duff CT, Sulla EM (2015) Measuring executive function in the differential diagnosis of attention-deficit/hyperactivity disorder: Does it really tell us anything? Appl Neuropsychol Child 4(3): 188-196.

43. Wu Z, Wang N, Qian Q Yang L, Qian Y, et al. (2014) Memory characteristic in boys with attention deficit/hyperactivity disorder comorbid learning disability. Zhonghua Yi Xue Za Zhi 94(22): 1701-1704.

44. George L, Ramirez J, Rivera P, Ojeda A (2014) C-24 Shared neuropsychological mechanisms of attention-deficit/hyperactivity disorder and eating disorders. Arch Clin Neuropsychol 29(6): 581-587.
45. Lin YT, Chen YC, Gau SS, Yeh TH, Fan HY, et al. (2016) Associations between allergic diseases and attention deficit hyperactivity/oppositional defiant disorders in children. Pediatr Res 80(4): 480-485.

46. Miyazaki C, Koyama M, Ota E, Swa T, Mlunde LB, et al. (2017) Allergic diseases in children with attention deficit hyperactivity disorder: A systematic review and meta-analysis. BMC Psychiatry 17(1): 120.

47. Jiang X, Shen C, Dai Y, Jiang F, Li S, et al. (2018) Early food allergy and respiratory allergy symptoms and attention-deficit/hyperactivity disorder in Chinese children: A cross-sectional study. Pediatr Allergy Immunol 29(4): 402-409.

48. Lee HK, Jeong JH, Kim NY, Park MH, Kim TW, et al. (2014) Sleep and cognitive problems in patients with attention deficit hyperactivity disorder. Neuropsychiatr Dis Treat 10: 1799-1805.

49. Metin B, Krebs RM, Wiersema JR, Verguts T, Gasthuys R, et al. (2015) Dysfunctional modulation of default mode network activity in attentiondeficit/hyperactivity disorder. J Abnorm Psychol 124(1): 208-214.

50. Hong SB, Zalesky A, Park S, Yang YH, Park MH, et al. (2015) COMT genotype affects brain white matter pathways in attention-deficit/ hyperactivity disorder. Hum Brain Mapp 36(1): 367-377.

51. Bailey $T$ (2010) Auditory pathways and processes: Implications for neuropsychological assessment and diagnosis of children and adolescents. Child Neuropsychol 16(6): 521-548.

52. Briese V, Stiete H, Stiete S (1997) Gestational diabetes--perinatal hyperinsulinism and postnatal developmental disorders Zentralbl Gynakol 119(7): 324-330.

53. Fernandez Jaen A, Cigudosa JC, Martin Fernandez Mayoralas D, Suela Rubio J, Fernandez Perrone AL, et al. (2014) Genetics applied to clinical practice in neurodevelopmental disorders. Rev Neurol 58 (Suppl) 1: S65-S70.

54. Getahun D, Rhoads GG, Demissie K, Lu SE, Quinn VP, et al. (2013) In utero exposure to ischemic-hypoxic conditions and attention-deficit/ hyperactivity disorder. Pediatrics 131(1): e53-e61.

55. Ghanizadeh A, Jafari P (2010) Risk factors of abuse of parents by their ADHD children. Eur Child Adolesc Psychiatry 19(1): 75-81.

56. Kachooei H, Daneshmand R, Dolatshahi B, Samadi R, Samiei M (2016) Attention-deficit/hyperactivity disorder and marital satisfaction: The preliminary roles of employment and Income. Iran J Psychiatry Behav Sci 10(2): e4012.

57. Kong L, Norstedt G, Schalling M, Gissler M Lavebratt C (2018) The risk of offspring psychiatric disorders in the setting of maternal obesity and diabetes. Pediatrics 142(3): e20180776.

58. Mina TH, Lahti M, Drake AJ, Räikkönen K, Minnis H, et al. (2017) Prenatal exposure to very severe maternal obesity is associated with adverse neuropsychiatric outcomes in children. Psychol Med 47(2): 353-362.

59. Porras Alonso E, Fornell Forcade J, Domingo Aznar L, Delgado Balufo D, García Campos D, et al. (1999) Auditory brain stem response and child attention deficit hyperactivity disorder. Acta Otorrinolaringol Esp 50(7): 567-570.

60. Scheinost D, Sinha R, Cross SN, Kwon SH, Sze G, et al. (2017) Does prenatal stress alter the developing connectome? Pediatr Res 81(1-2): 214-226.

61. Silva D, Colvin L, Hagemann E, Bower C (2014) Environmental risk factors by gender associated with attention-deficit/hyperactivity disorder. Pediatrics 133(1): e14-e22.

62. Smeyers P (1999) Studies of evoked potentials in children with the syndrome of attention deficit and hyperactivity. Rev Neurol 28 (Suppl) 2: S173-S176.

63. Weissenberger S, Ptacek R, Klicperova Baker M, Erman A, Schonova K, et al. (2017) ADHD, lifestyles and comorbidities: A call for an holistic 
perspective - from medical to societal intervening factors. Front Psychol 8: 454.

\section{ISSN: 2574-1241}

DOI: 10.26717/BJSTR.2018.12.002228

Orlando J Castejón. Biomed J Sci \& Tech Res

(c) (P) This work is licensed under Creative

Submission Link: https://biomedres.us/submit-manuscript.php
64. Zhu T, Gan J, Huang J, Li Y, Qu Y, et al. (2016) Association between perinatal hypoxic-ischemic conditions and attention-deficit/hyperactivity disorder: A meta-analysis. J Child Neurol 31(10): 1235-1244.

\begin{tabular}{ll} 
BIOMEDICAL & Assets of Publishing with us \\
RESEARCHES & - Global archiving of articles \\
& - Immediate, unrestricted online access \\
\hline
\end{tabular}

